\title{
SUSCEPTIBILIDAD DE TETRANYCHUS URTICAE KOCH (ACARI: TETRANYCHIDAE) A LOS PLAGUICIDAS CLORFENAPIR Y ABAMECTINA EN CONDICIONES DE LABORATORIO
}

\author{
EFFICACY OF CHLORFENAPYR AND ABAMECTIN TO CONTROL \\ OF TETRANYCHUS URTICAE KOCH (ACARI: TETRANYCHIDAE)
}

\author{
Carlos Vásquez ${ }^{1}$; María C. Ceballos ${ }^{1}$
}

\begin{abstract}
RESUMEN
Se evaluó la eficiencia de control del Clorfenapir y Abamectina sobre una población de Tetranychus urticae bajo condiciones de laboratorio $\left(27 \pm 2{ }^{\circ} \mathrm{C}, 70 \pm 10 \%\right.$ de H.R.). La población del ácaro fue colectada en plantas de tomate (Lycopersicon esculentum L.) en la población de "Guadalupe", Municipio Jiménez, Estado Lara. En el laboratorio se evaluó el efecto de diferentes concentraciones de Clorfenapir (37, 75, 150, 300, 600, 1200 y 2400 ppm) y Abamectina (1,6;2,3; 3,2; 4,5; 6,3; 8,8 y 12,4 ppm) usando la técnica de inmersión del disco de hoja. Las $\mathrm{CL}_{50}$ estimadas para Clorfenapir y Abamectina fueron 59,34 y 1,50 ppm, respectivamente y son menores a la dosis recomendada para las aplicaciones en campo. El Clorfenapir mostró ser más efectivo en el control de T. urticae, sin embargo su efecto sobre la fauna benéfica debe ser evaluado. Es necesario realizar estudios de campo para validar los resultados obtenidos en la presente investigación.
\end{abstract}

Palabras clave: plaguicidas, ácaro de las dos manchas, mortalidad.

\section{ABSTRACT}

Efficacy of Chlorfenapyr and Abamectin to control a Tetranychus urticae population was determined under laboratory conditions $\left(27 \pm 2{ }^{\circ} \mathrm{C}, 70 \pm 10 \%\right.$ R.H.). The population was collected in tomato plants (Lycopersicon esculentum L.) in "Guadalupe", Jimenez County, Lara state. At the laboratory effect of different Chlorfenapyr (37, 75, 150, 300, 600, 1200 and 2400 ppm) and Abamectin $\left(1,6 ; 2,3 ; 3,2 ; 4,5 ; 6,3 ; 8,8\right.$ and 12,4 ppm) concentrations was evaluated using disk leaves immersion technique. $L C_{50}$ values obtained for Chlorfenapyr and Abamectin were 59.34 and $1.50 \mathrm{ppm}$, respectively and they are lower than recommended field dose. Chlorfenapyr showed to be more effective in to control $\mathrm{T}$. urticae, however effect on benefical fauna should be evaluated. Field studies to validate results herein obtained are required.

Key words: pesticide, two spotted spider mite, concentrations.

\section{INTRODUCCIÓN}

El ácaro de las dos manchas Tetranychus urticae Koch, 1836, es considerado una plaga severa en numerosos cultivos de importancia económica a nivel mundial (Jeppson et al., 1975, Bolland et al., 1998). Los síntomas típicos producidos por la alimentación de los tetraníquidos en la planta hospedera incluyen alteraciones mecánicas y bioquímicas, las cuales se manifiestan como cambios en la coloración de la hoja, reducción de la tasa de crecimiento, retraso de la floración y disminución en la producción de frutos (Tomczyk y Kropeznska, 1985).

Debido al impacto económico producido por el ataque de T. urticae en diferentes sistemas de producción agrícola, la estrategia de control ha sido casi exclusivamente basada en el uso de agroquímicos, sin embargo su efectividad ha sido variable 1 Universidad Centroccidental Lisandro Alvarado. Decanato de Agronomía. Departamento de Ciencias Biológicas. Barquisimeto,
Estado Lara. Venezuela. E-mail: carlosvasquez@ucla.edu.ve 
(Ay et al., 2005). Actualmente existe una variedad de productos con efecto acaricida e insecticida con novedosos mecanismos de acción, entre los cuales la Abamectina y el Clorfenapir se incluyen entre los productos de acción acaricida más promisorios, debido a que mantienen las poblaciones de T. urticae por debajo del umbral económico (Ay et al., 2005, Sato et al., 2005).

Las avermectinas son un grupo de lactonas macrocíclicas con efecto insecticida, acaricida y nematicida de nueva generación (Campos et al., 1996). A pesar de que este grupo de plaguicidas no ha sido ampliamente usado, algunos estudios han reportado desarrollo de bajos a moderados niveles de resistencia en poblaciones de T. urticae colectadas en pera (Pyrus sp.), mientras que las poblaciones de Tetranychus mcdanieli McGregor, 1931, mostraron ser mucho más resistentes a este producto (Beers et al., 1998). Por otra parte, Lin et al. (2003) demostraron que el índice de heredabilidad de la resistencia de Tetranychus cinnabarinus (Boisduval) a la Abamectina era comparativamente mayor $(0,15)$ que para Pyridaben $(0,03)$.

Con relación al Clorfenapir, este corresponde a un proinsecticida (insecticida que se convierte a su forma activa mediante las enzimas del insecto, inhibiendo la fosforilación oxidativa en las mitocondrias celulares) usado contra un amplio rango de especies plaga entre las que se incluyen ácaros tetraníquidos, eriófidos y tarsonémidos, demostrando ser efectivo para el control de plagas catalogadas como resistentes (Leonard, 2000). Hasta el presente, pocos casos han sido documentados sobre el desarrollo de resistencia de ácaros fitófagos a este producto. Uesugi et al. (2002) detectaron que la resistencia en T. urticae al Clorfenapir era de tipo dominante y además encontraron que debido a la cercanía de los genes resistentes esto podría provocar el desarrollo de resistencia cruzada en cultivos con frecuentes aplicaciones del producto. Sin embargo, Stumpf y Nauen (2001) no observaron desarrollo de resistencia en cuatro poblaciones de T. urticae cuando fueron tratadas con Clorfenapir.

En la Depresión de Quibor, Estado Lara, el control de ácaros Tetranychidae se hace mediante el uso de productos a base de Abamectina con alta frecuencia de aplicación, lo que podría conllevar al desarrollo de resistencia a corto plazo, considerando la alta tasa de reproducción y el corto ciclo de vida de esta plaga (Croft y van den Baan, 1998). Por ello, en la presente investigación se evaluó la susceptibilidad de una población de T. urticae a diferentes concentraciones de Clorfenapir y Abamectina a fin de ofrecer a productores de la región una alternativa segura de control con el uso de agroquímicos.

\section{MATERIALES Y MÉTODOS}

\section{A. COLECTA Y DETERMINACIÓN DEL ÁCARO}

Los ácaros fueron colectados de plantas de tomate en la población de Guadalupe, Municipio Jiménez, Estado Lara, donde previamente se ha usado Vertimec $^{\mathrm{MR}}$ para el manejo de poblaciones de diferentes especies plaga. Para el muestreo se tomaron hojas provenientes de cinco plantas seleccionadas al azar mostrando síntomas de ataque de tetraníquidos. Las muestras de hojas fueron colocadas en bolsas tipo clic recubiertas internamente con papel absorbente y transportadas al laboratorio en una cava refrigerada para evitar la muerte de los ácaros por las altas temperaturas del campo.

En el laboratorio, las hojas fueron examinadas bajo el aumento de una lupa estereoscópica Leica MS-5 a 40X y los especímenes fueron montados en láminas microscópicas usando Líquido de Hoyer, las cuales fueron posteriormente secadas en estufa a 40 ${ }^{\circ} \mathrm{C}$ durante tres días. La determinación del género se hizo utilizando la clave taxonómica de Gutiérrez (1985) y la especie fue determinada mediante comparación de la morfología del aedeagus con las ilustraciones dadas por Ochoa et al. (1994).

\section{B. DETERMINACIÓN DE LA \\ SUSCEPTIBILIDAD DE T. URTICAE A DIFERENTES CONCENTRACIONES DE CLORFENAPIR Y ABAMECTINA}

Se evaluó el efecto de diferentes concentraciones de Clorfenapir (Sunfire ${ }^{\mathrm{MR}} 240$ g. $^{-1}$ ) y Abamectina (Vertimec $^{\mathrm{MR}} 1,8 \mathrm{~g} .100 \mathrm{~m} \mathrm{l}^{-1}$ ) sobre la mortalidad de T. urticae. Las dosis fueron seleccionadas en función a la dosis comercial recomendada por el fabricante $(0,5$ a 0,71 i.a./ha). Para el Clorfenapir se usaron 37, 75, 150, 300, 600, 1200 y 2400 ppm de i.a., mientras que para Abamectina se usaron 1,$6 ; 2,3 ; 3,2 ; 4,5 ; 6,3 ; 8,8$ y 12,4 ppm.

La susceptibilidad de la población de T. urticae al Clorfenapir y Abamectina fue determinada siguiendo la técnica de inmersión de discos de hoja (Cowles et al. 2000). Esta consistió en sumergir discos de hoja de Phaseolus lunatus (Leguminosae) (6 cm de 
diámetro) durante cinco segundos en cada una de las concentraciones evaluadas. Posteriormente los discos de hoja fueron extraídos, sacudidos suavemente para eliminar el exceso de líquido y secados a temperatura ambiente $\left(27 \pm 2{ }^{\circ} \mathrm{C}, 70 \pm 10 \%\right.$ de H.R.) durante 1 hora. Posteriormente los discos de hoja fueron transferidos a unidades de cría tal como fue descrito por Helle y Overmeer (1985). Cada unidad de cría o arena consistió de una cápsula de Petri ( $9 \mathrm{~cm}$ de diámetro x $1,5 \mathrm{~cm}$ de altura), dentro de la cual se ajustó una almohadilla circular de poliuretano de $1 \mathrm{~cm}$ de espesor. Seguidamente, un disco de hoja fue colocado sobre la almohadilla con el envés hacia arriba y fijado con una banda de algodón humedecida de $1 \mathrm{~cm}$ de ancho con el fin de evitar el escape de los ácaros y mantener la turgencia de los discos de hojas.

Se prepararon 15 unidades de cría para cada una de las concentraciones a evaluar de cada producto, sobre las cuales se colocaron 15 hembras de T. urticae de dos a tres días de edad. Se utilizó agua como tratamiento control. Las unidades de cría fueron examinadas bajo aumento de una lupa estereoscópica $(40 \mathrm{X})$ para determinar la motilidad de las hembras a las 24 y 72 horas después del tratamiento para Clorfenapir y Abamectina, respectivamente. Los experimentos fueron repetidos tres veces y los resultados obtenidos fueron agrupados de acuerdo a los tratamientos. Se consideró como muerta una hembra que no presentó ningún tipo de movimiento al contacto con un pincel (000).

Los valores de $\mathrm{CL}_{50}, \mathrm{CL}_{95}$ para cada uno de los productos evaluados fueron sometidos a análisis Probit (Stat Plus 2005).

\section{RESULTADOS Y DISCUSIÓN}

Los resultados del análisis Probit sobre los datos de concentración-mortalidad para T. urticae expuestos a Clorfenapir y Abamectina se muestran en el Cuadro 1. Los valores de la $\mathrm{CL}_{50}$ obtenidos tanto para Clorfenapir como para Abamectina son inferiores a las dosis recomendadas en campo (300 y $4,5 \mathrm{ppm}$, respectivamente), lo que permite inferir que la población bajo estudio pudiera ser controlada aún con bajas concentraciones de estos productos comerciales. Con relación al Clorfenapir, la población bajo estudio mostró ser susceptible aun cuando se usaron concentraciones muy bajas posiblemente porque este ingrediente activo nunca había sido utilizado en el control en la zona de muestreo. Uesugi et al. (2002) consiguieron valores de $\mathrm{CL}_{50}$ para el Clorfenapir de 2.130 ppm para razas resistentes de T. urticae $\left(\mathrm{F}_{1}\right)$, mientras que para aquellas susceptibles fue de 4,77 ppm. Sin embargo, los datos deben convalidarse con estudios de campo bajo diferentes condiciones ambientales.

De manera similar, se encontró que el valor de la $\mathrm{CL}_{50}$ de Abamectina obtenida para la población bajo estudio está por debajo de la dosis de campo (4,5 ppm). Campos et al. (1995) encontraron que la mortalidad de las poblaciones susceptibles de T. urticae alcanzó niveles que variaron entre $30 \mathrm{y}$ $97 \%$ con concentraciones entre 0,03 y 1 ppm, mientras que concentraciones entre 3 y 10 ppm lograron baja tasa de mortalidad en poblaciones consideradas como resistentes. Campos et al. (1996) demostraron que el desarrollo de la resistencia a la Abamectina está relacionado con la frecuencia de aplicación del producto y encontraron factores de resistencia que oscilaron entre 0,5 y 175 en un bioensayo de un día. En poblaciones de T. urticae provenientes de Holanda la resistencia se desarrolló cuando habían sido expuestas por más de tres veces.año ${ }^{-1}$ durante dos años, mientras que en las poblaciones provenientes de California este fenómeno fue detectado cuando la frecuencia de aplicación fue de más de seis veces.año ${ }^{-1}$ durante cuatro años consecutivos. Los resultados obtenidos en el presente estudio

Cuadro 1

Análisis Probit de los datos de concentración-mortalidad para T. urticae expuesto a varias concentraciones de Clorfenapir y Abamectina

\begin{tabular}{|c|c|c|c|c|c|}
\hline & $\mathbf{N}$ & Pendiente & $\begin{array}{c}\mathbf{C} \mathbf{L}_{50}(\mathbf{p p m}) \\
(\mathbf{I C} 95 \%)\end{array}$ & $\mathbf{C L}_{95}(\mathbf{p p m})$ & $\begin{array}{c}\chi^{2} \\
(\mathrm{~g} . \mathrm{l}=5)\end{array}$ \\
\hline Clorfenapir & 120 & $2,33 \pm 1,1746$ & $59,34(0-150,97)$ & 301,30 & 0,183 \\
\hline Abamectina & 120 & $4,43 \pm 3,1822$ & $1,50(0,72-3,13)$ & 3,53 & 0,4064 \\
\hline
\end{tabular}


permiten inferir sobre la posibilidad de desarrollo de resistencia en las poblaciones de T. urticae provenientes de la localidad de Guadalupe (Municipio Jiménez, Estado Lara) dada la alta frecuencia de aplicación y la fuerte presión de selección ejercida por las altas dosis aplicadas.

Con relación al efecto de la concentración se encontró que al final del período de evaluación el Clorfenapir mostró mayor eficiencia de control (Fig. 1A). La totalidad de los individuos evaluados aparecieron muertos por efecto del producto cuando se usaron las concentraciones de $150 \mathrm{ppm}$, la cual representa la mitad de la concentración recomendada por el fabricante. Resultados similares fueron obtenidos por Cote (2001) quien encontró que el Clorfenapir produjo mejor control sobre poblaciones de T. urticae al ser comparado con Abamectina, incluso en poblaciones de ácaros que fueron transferidos a plantas que habían sido asperjadas con el producto 14 días antes de la infestación. Adicionalmente, este autor encontró que los residuos de Clorfenapir produjeron 55\% de mortalidad de T. urticae después de dos semanas comparado con $6 \%$ de mortalidad en el control. Sin embargo, Lin et al. (2003) determinaron que las poblaciones de Tetranychus cinnabarinus (Boisduval) incrementaron 3,7 veces su resistencia a la Abamectina en 16 generaciones consecutivas mantenidas en el laboratorio.

A pesar de la alta efectividad mostrada por el Clorfenapir, su uso en agroecosistemas debe ser hecho bajo un sistema adecuado de supervisión puesto que este producto muestra efectos negativos sobre la fauna benéfica. Cote (2001) encontró que este producto causaba alto porcentaje de mortalidad en Phytoseiulus persimnilis Athias-Henriot, por lo que el autor no recomienda su inclusión en programas que incluyan este depredador como controlador biológico. De manera similar, Leonard (2000) evaluó el efecto de Clorfenapir sobre los ácaros fitoseídos y encontró que este producto ejerció un efecto negativo sobre las poblaciones de Typhlodromus pyri, pero
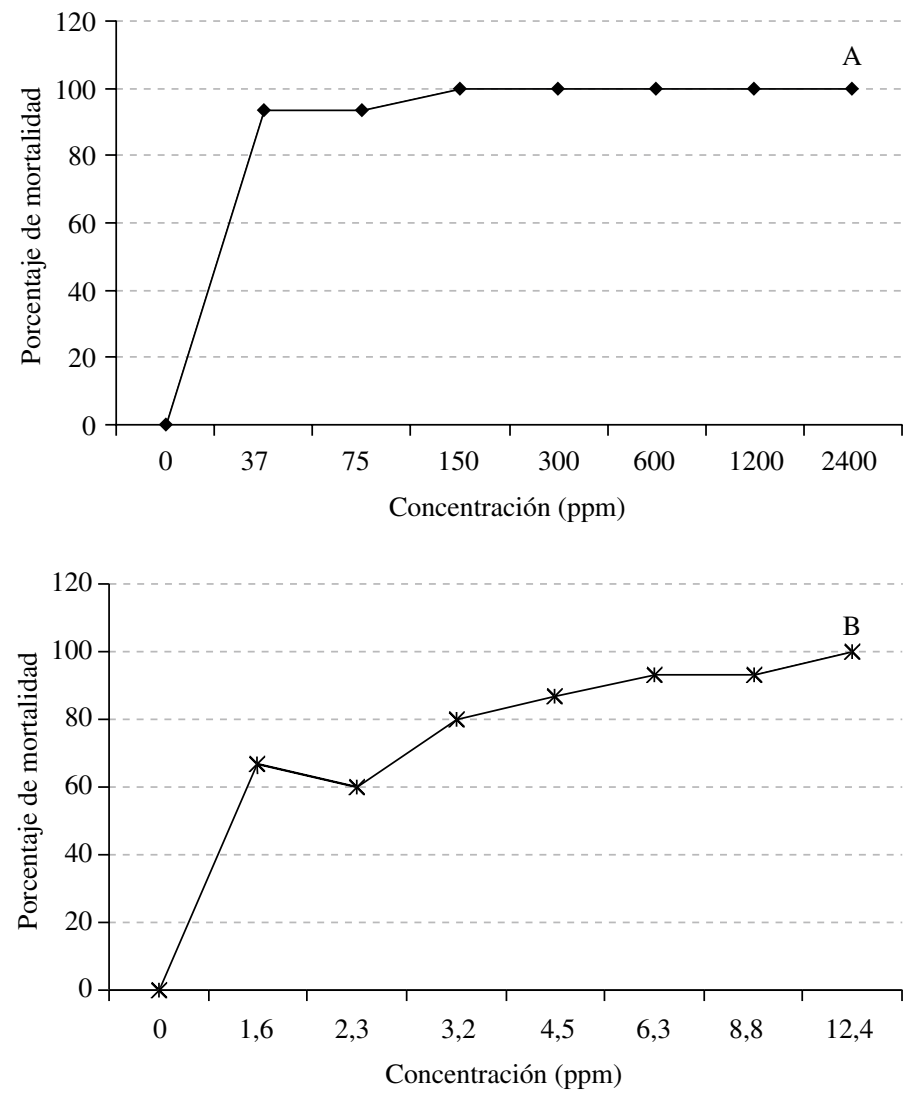

Figura 1. Curva de dosis-mortalidad de una población de T. urticae tratada con Clorfenapir (A) o Abamectina (B) 
no afectó las poblaciones de Amblyseius aberrans. De manera similar, se encontró que la mortalidad de la población de $T$. urticae estuvo en relación directa con el incremento de la concentración de Abamectina (Fig. 1B). Las diferencias observadas en las curvas de dosis-mortalidad evidenciaron variaciones genéticas entre los individuos de la población (Ay et al., 2005).

La mortalidad alcanzada por la aplicación de Abamectina a 1,6 ppm fue ligeramente superior a la observada con 2,3 ppm, luego mostró una tendencia a incrementarse hasta alcanzar el máximo control con la mayor concentración. Ay et al. (2005) demostraron que las poblaciones de T. urticae provenientes de Keciborlu-Isparta mostraron acentuada pérdida de la susceptibilidad contra la Abamectina que el resto de las poblaciones probadas. Adicionalmente estos autores aseguraron, basados en el valor de la proporción de resistencia $(3,9)$, que esta población tiene mayor probabilidad para el desarrollo de resistencia, considerando la

\section{LITERATURA CITADA}

AY, R.; SÖKELI, E; KARACA I.; GURKAN M.O. 2005. Response to some acaricides of two-spotted spider mites (Tetranychus urticae Koch) from protected vegetable in Isparta. Turkish Journal of Agriculture and Forestry, 29: 165-171.

BEERS, E.; RIEDL H.; DUNLEY J.E. 1998. Resistance to abamectin and reversion to susceptibility to fenbutatin oxide in spider mites (Acari: Tetranychidae) populations in the Pacific Northwest. Journal of Economical Entomology, 91: 352-360.

BOLLAND, H.R.; GUTIÉRREZ J; FLETCHMANN C.H.W. 1998. World Catalogue of the Spider Mite Family (Acari: Tetranychidae). Koninklijke Brill NV, Leiden, The Netherlands. Pp. XX.

CAMPOS, F.; KRUPA D.; DYBAS R. 1995. Susceptibility of twospotted spider mites (Acari: Tetranychidae) populations in California to abamectin. Journal of Economical Entomology, 88: 225-231.

CAMPOS, F.; KRUPA D.; DYBAS. R. 1996. Susceptibility of populations of twospotted spider mites (Acari: Tetranychidae) from Florida, Holland, and the Canary Island to abamectin and characterization of abamectin resistance. Journal of Economical Entomology, 89: 594-601.

COTE, K. 2001. Using selected acaricides to manipulate Tetranychus urticae Koch populations in order to enhance biological control provided by phytoseiid mites. Tesis de Magister. Virginia Polytechnical Institute and State University. 107 pp.

COWLES, R.S.; COWLES E.A.; MCDERMOTT A.M.; RAMOUTAR D. 2000. "Inert" formulation ingredients with activity: toxicity of Trisiloxane surfactant solutions frecuencia de aplicación para mantener las poblaciones por debajo del umbral económico aunado a la alta tasa reproductiva y ciclo de vida corto de estos ácaros (Nauen et al., 2001).

\section{CONCLUSIONES Y RECOMENDACIONES}

El Clorfenapir mostró ser más efectivo que la Abamectina, dado que las menores concentraciones del producto lograron control de hasta $100 \%$ en la población evaluada. Sin embargo, se recomienda realizar estudios de laboratorio que incluyan la comparación entre poblaciones colectadas en campo y susceptibles de modo de monitorear el posible desarrollo de resistencia, puesto que la alta frecuencia de aplicación y altas dosis usadas en campos hortícolas de la Depresión de Quibor permiten inferir sobre la posibilidad de desarrollo de resistencia a esta molécula en corto tiempo.

to twospotted spider mites (Acari: Tetranychidae). Journal of Economical Entomology, 2: 180-188.

CROFT, B.A.; VAN DE BAAN, H. E. 1988. Ecological and genetics factors influencing evolution of pesticides resistance in Tetranychid and Phytoseiid mites. Experimental and Applied Acarology, 4: 277-300.

GUTIERREZ, J. 1985. Systematics. In: Helle W. y Sabelis M. Spider Mites: Their Biology, Natural Enemies and Control. Elsevier Science Publishers B. V., Amsterdam, The Netherlands. Pp. Xx-yy.

HELLE, W.; OVERMEER, W.P.J. 1985. Toxicological Test Methods. In: Helle W. and Sabelis M. Spider Mites: Their Biology, Natural Enemies and Control. Vol 1A. Elsevier Science Publishing Co., Amsterdam, The Netherlands. pp. 391-395.

JEPPSON, H; KEIFER, H; BAKER, E. 1975. Mites Injurious to Economic Plants. Univ. of California. Press. Riverside. $614 \mathrm{p}$.

LEONARD, P.K. 2000. Chlorfenapyr, a novel IPM compatible resistance management tool for fruit production. Acta Horticulturae, 525: 257-275

LIN, H.; ZHAO Z.; DENG X.; WANG J.; LIU H. 2003. Resistance risk assessment: realized heritability of resistance to methrin, abamectin, pyridaben and their mixtures in the spider mite, Tetranychus cinnabarinus. International Journal of Pest Management, 49: 271-274.

NAUEN, R.; STUMPF N.; ELBERT A.; ZEBITZ C.P.W.; KRAUS W. 2001. Acaricide toxicity and resistance in larvae of different strains of Tetranychus urticae and Panonychus ulmi (Acari: Tetranychidae). Pest Management Science, 57: 253-261. 
OCHOA, R; AGUILAR, H; VARGAS, C. 1994. Phytophagous Mites of Central America: An Illustrated Guide. CATIE, Turrialba, Costa Rica. 234 pp.

SATO M.E., SILVA DA, M.; RAGA, A; SOUZA DE, M.F. 2005. Abamectin resistance in Tetranychus urticae Koch (Acari: Tetranychidae): selection, cross-resistance and stability of resistance. Neotropical Entomology, 34 (6): 991-998.

STAT PLUS PARA WINDOWS. Versión 3.5. Alexey Simachov Copyright $\odot$, 2001-2005.

STUMPF. N.; R. NAUEN. 2001. Cross-resistance, inheritance, and biochemistry of mitochondrial electron transport inhibitor-acaricide resistance in Tetranychus urticae (Acari: Tetranychidae). Journal of Economical Entomology, 94: 1577-1583.

TOMCZYK, A.; KROPCZNSKA, D. 1985. Effects on the Host Plant. In: Helle W. y Sabelis M. 1985. Spider Mites: Their Biology, Natural Enemies and Control. Vol 1A. Elsevier Science Publishing Co., Amsterdam, The Netherlands. Pp. Xx-yy.

UESUGI, R.; GOKA K.; OSAKABE, M.H. 2002. Genetics basis of resistance to chlorfenapyr and etoxazole in the two-spotted spider mite (Acari: Tetranychidae). Journal of Economical Entomology, 95: 1267-1274. 